\title{
Histiocytose $\mathrm{X}$ et adénocarcinome broncho-pulmonaire : rare coexistence
}

\author{
Akýn Kaya $M D^{1}$, Ýsmail Savap $M D^{1}$, Elif Pen $M D^{1}$, Serpil Dizbay Sak $M D^{2}$, \\ Adem Güngör $M D^{3}$, Uður Gönüllü MD ${ }^{1}$
}

\begin{abstract}
A Kaya, I Savap, E Pen, SD Sak, A Güngör, U Gönüllü. Histiocytose $\mathrm{X}$ et adénocarcinome broncho-pulmonaire : rare coexistence. Can Respir J 2002;9(6):431-432.
\end{abstract}

L'association de l'histiocytose X du poumon et du cancer bronchopulmonaire s'observe rarement, mais la fréquence de ce type de cancer chez ces patients est plus élevée que dans la population normale. Voici le cas d'une patiente âgée de 28 ans, tabagique à 10 paquets-années, qui s'est adressée à notre service de pneumologie pour de la toux et des hémoptysies. La radiographie du thorax a révélé des kystes bilatéraux et une opacité dans la partie supérieure du champ pulmonaire droit et la tomodensitométrie thoracique a montré de multiples kystes dispersés dans les deux champs pulmonaires et une masse aux contours irréguliers, de $4 \mathrm{~cm}$ de diamètre, dans le lobe supérieur droit. L'examen cytologique des prélèvements du lobe supérieur droit, effectués par lavage bronchique a permis la pose du diagnostic d'adénocarcinome broncho-pulmonaire. Comme il n'y avait pas de métastases à distance, la thoracotomie a été décidée. Nous avons donc procédé à une lobectomie supérieure et à une résection cunéiforme ( "wedge ») du segment supérieur du lobe inférieur droit, territoires envahis par la tumeur. L'examen histologique du tissu tumoral a confirmé le diagnostic d'adénocarcinome et la biopsie du tissu pulmonaire non tumoral a révélé l'existence de l'histiocytose X. Il se pourrait que l'apparition d'une néoplasie sur fibrose pulmonaire soit un épiphénomène par rapport au cancer broncho-pulmonaire lié à la consommation de tabac chez les patients atteints d'histiocytose $\mathrm{X}$ du poumon. Il est intéressant de souligner que les diagnostics d'histiocytose $\mathrm{X}$ et de cancer broncho-pulmonaire ont été posés en même temps, car le cancer aurait pu apparaittre bien des années après la pose du diagnostic d'histiocytose, même si la patiente fumait. Les hémoptysies qui se rencontrent chez $5 \%$ des patients atteints d'histiocytose $\mathrm{X}$ peuvent être évocatrices du cancer. La jeune femme, fumeuse, a consulté pour des hémoptysies; il s'agit là d'un cas particulièrement rare d'histiocytose $\mathrm{X}$ du poumon associée à un cancer broncho-pulmonaire, dont la pathogenèse n'est pas claire. Il importe donc de savoir que le tabagisme peut avoir de graves conséquences même pour les jeunes gens.

Mots clés: Adenocarcinome bronchopulmonaire; Hemoptysie; Histiocytose X

\section{Histiocytosis $\mathrm{X}$ and bronchopulmonary adenocarcinoma: $\mathrm{A}$ rare coexistence}

There exists a rarely observed association between pulmonary histiocytosis $\mathrm{X}$ and bronchopulmonary cancer. However, the frequency of bronchopulmonary cancer in these patients is higher than in the general population. A 28-year-old patient who currently smokes ten packs of cigarettes a year came to our department of pneumology with complains of cough and hemoptysis. An x-ray of the thorax revealed bilateral cysts and a shadow in the upper part of the right pulmonary field. In addition, a chest tomography showed multiple cysts dispersed throughout the two pulmonary fields and an irregular mass with a diameter of four centimetres in the upper right lobe. Bronchopulmonary adenocarcinoma was diagnosed during a cytologic exam of the bronchial washing. We decided to perform a thoracotomy on the patient, since there was no far metastasis. An upper lobectomy and wedge resection of the upper segment of the lower right lobe, which had been invaded by the tumour, were performed.

Continued on next page

\footnotetext{
${ }^{1}$ Service de Pneumologie de l'Hôpital Universitaire d'Ankara, ${ }^{2}$ Département de Pathologie de l'Hôpital Universitaire d'Ankara, ${ }^{3}$ Service de la Chirurgie du Thorax de l'Hôpital Universitaire d'Ankara, Ankara, Türkiye L'adresse de correspondance: Docteur Elif pen, And Sokak 14/6 Çankaya/Ankara, 06680, Türkiye. Téléphone +90-362-30-30 ext 6510, fax+90-312-319-00 46, e-mail elifsen2001@yahoo.com
} 
Histology confirmed the diagnosis of adenocarcinoma. A pulmonary biopsy was carried out on the tumour-free site and showed the presence of histiocytosis X. There is a hypothesis that a neoplasm developed on the pulmonary fibrosis could be an epiphenomenon of bronchopulmonary cancer in patients who smoke and have pulmonary histiocytosis X. It is interesting to note that histiocytosis $\mathrm{X}$ and bronchopulmonary cancer were diagnosed at the same time, since the bronchopulmonary cancer may have occurred within a few years following the diagnosis of histiocytosis $\mathrm{X}$, even if she was a smoker. Hemoptysis, which is found in $5 \%$ of patients with histiocytosis $\mathrm{X}$, may suggest cancer. This young patient, a smoker, who complained of hemoptysis, is a particularly rare case of the association between pulmonary histiocytosis $\mathrm{X}$ and bronchopulmonary cancer whose pathogenesis is not clear cut. It is thus important to note that smoking can have major consequences, even in young people.
T 'association de l'histiocytose $\mathrm{X}$ du poumon et du cancer Lbroncho-pulmonaire s'observe rarement d'après la littérature (1) et la pose simultanée des deux diagnostics est extrêmement rare.

Mme D., née en 1973, tabagique à 10 paquets-années, s'est adressée à notre service de pneumologie pour de la toux et des hémoptysies. La toux avait commencé deux mois et demi auparavant et les hémoptysies étaient apparues au cours des derniers jours. L'examen physique s'est avéré tout à fait normal. La radiographie du thorax a révélé des kystes bilatéraux et une opacité dans la partie supérieure du champ pulmonaire droit. La tomodensitométrie thoracique (Figure 1) a montré de multiples kystes dispersés dans les deux champs pulmonaires et une masse aux contours irréguliers, de $4 \mathrm{~cm}$ de diamètre, dans le lobe supérieur droit. L'examen cytologique des prélèvements du lobe supérieur droit, effectués par lavage bronchique a permis la pose du diagnostic d'adénocarcinome broncho-pulmonaire. Les examens tomodensitométriques de l'abdomen et de l'encéphale ainsi que la scintigraphie osseuse se sont révélés normaux; la thoracotomie droite a donc été décidée. Nous avons procédé à une lobectomie supérieure et à une résec-

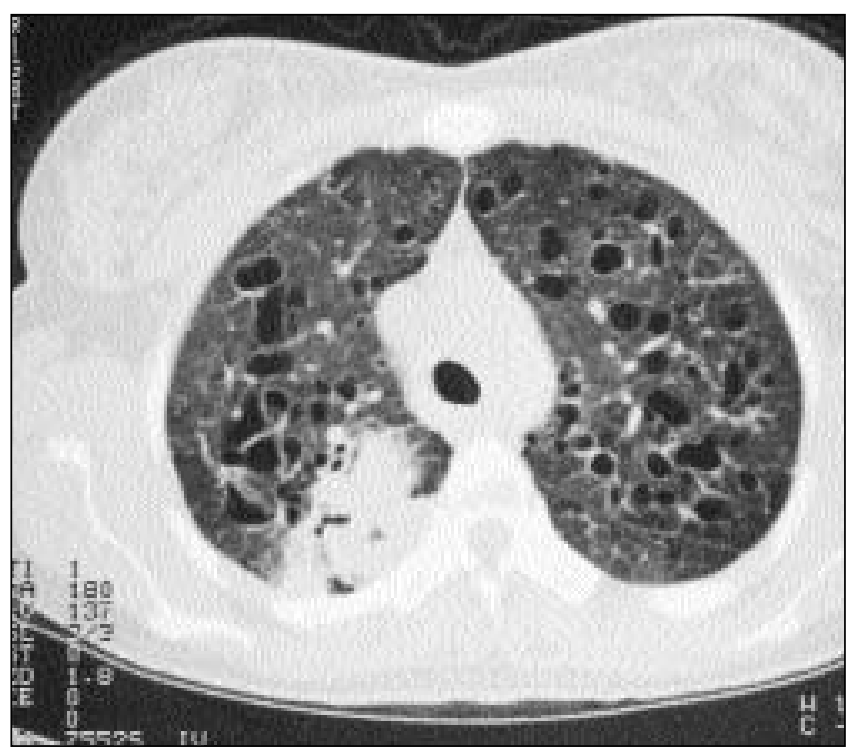

Figure 1) Tomodensitométrie thoracique sur laquelle on voit de multiples kystes dispersés dans les deux champs pulmonaires et la masse aux contours irréguliers dans le lobe supérieur droit tion cunéiforme ( «wedge ») du segment supérieur du lobe inférieur droit, territoires envahis par la tumeur.

L'examen histologique du tissu tumoral a confirmé le diagnostic d'adénocarcinome et la biopsie du tissu pulmonaire non tumoral a révélé l'existence de l'histiocytose X. La patiente a cessé de fumer après l'opération et elle est suivie régulièrement.

Même si l'association de l'histiocytose X du poumon et du cancer broncho-pulmonaire est faible, on admet que la fréquence de ce type de cancer chez ces patients est plus élevée que dans la population normale (2). Selon Sadoun et coll., le cancer broncho-pulmonaire se manifesterait environ 10,5 ans après la pose du diagnostic d'histiocytose X (1). Deux facteurs sont responsables de cette association : le tabagisme et la fibrose pulmonaire. D'après la littérature, il se peut que le rôle du tabagisme soit plus important (2) et que l'apparition d'une néoplasie sur fibrose pulmonaire soit un épiphénomène par rapport au cancer bronchopulmonaire lié à la consommation de tabac (3). Le cancer broncho-pulmonaire peut être cause de morbidité et de mortalité chez les patients d'âge moyen, atteints d'histiocytose $\mathrm{X}$ (1). La patiente en question présente des particula-rités notables en ce qui concerne cette association. Le diagnostic d'histiocytose X du poumon n'a pas précédé l'apparition du cancer. L'augmentation de l'incidence du cancer bronchopulmonaire secondaire au tabagisme chez les femmes est certes réalité, mais la patiente est trop jeune pour cela, bien qu'elle fumait l'équivalent de 10 paquets-années.

Les hémoptysies se rencontrent chez $5 \%$ des patients atteints d'histiocytose $\mathrm{X}$ et sont évocatrices du cancer (2) mais, dans le présent cas, elles étaient révélatrices des deux affections, d'après le cliché du thorax. La jeune femme, fumeuse, a consulté pour des hémoptysies; il s'agit là d'un cas particulièrement rare d'histiocytose $\mathrm{X}$ du poumon associée à un cancer broncho-pulmonaire, dont la pathogenèse n'est pas claire. Il importe donc de savoir que le tabagisme peut avoir de graves conséquences même pour les jeunes gens.

\section{RÉFÉRENCES}

1. Sadoun D, Vaylet F, Valeyre D, et al. Bronchogenic carcinoma in patients with pulmonary histiocytosis X. Chest 1992;101:1610-3.

2. Vassallo R, Ryu JH, Colby TV, Hartman T, Limper AH. Pulmonary Langerhans' cell histiocytosis.Med Prog 2000;342:1969-78.

3. Lombard CM, Medeiros LJ, Colby TV. Pulmonary histiocytosis X and carcinoma. Arch Pathol Lab Med 1987;111:339-41. 


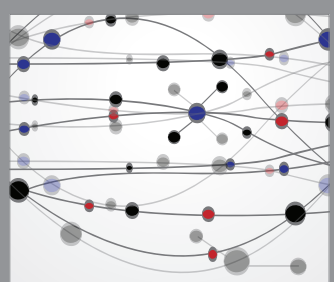

The Scientific World Journal
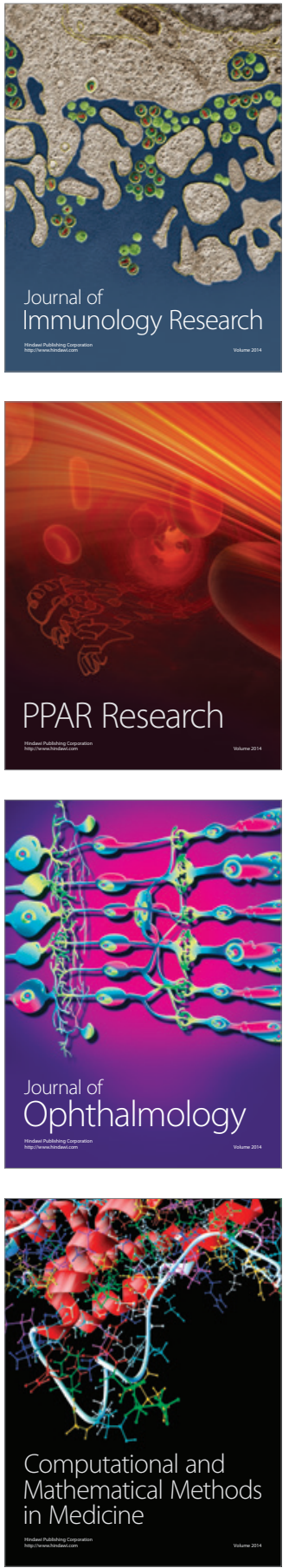

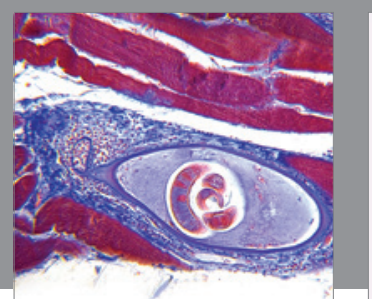

Gastroenterology Research and Practice

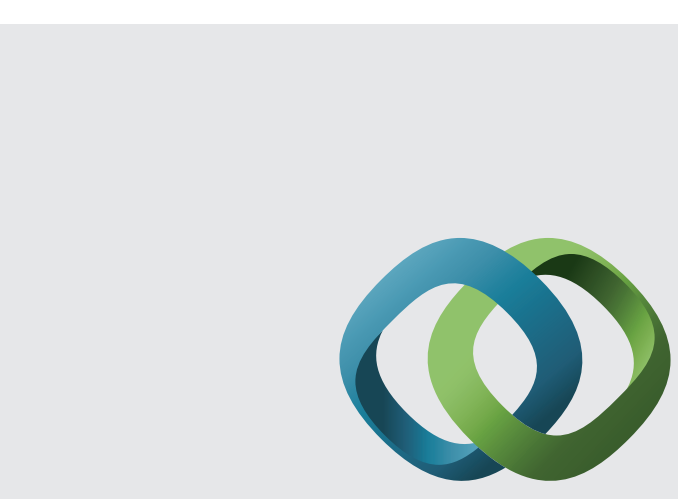

\section{Hindawi}

Submit your manuscripts at

http://www.hindawi.com
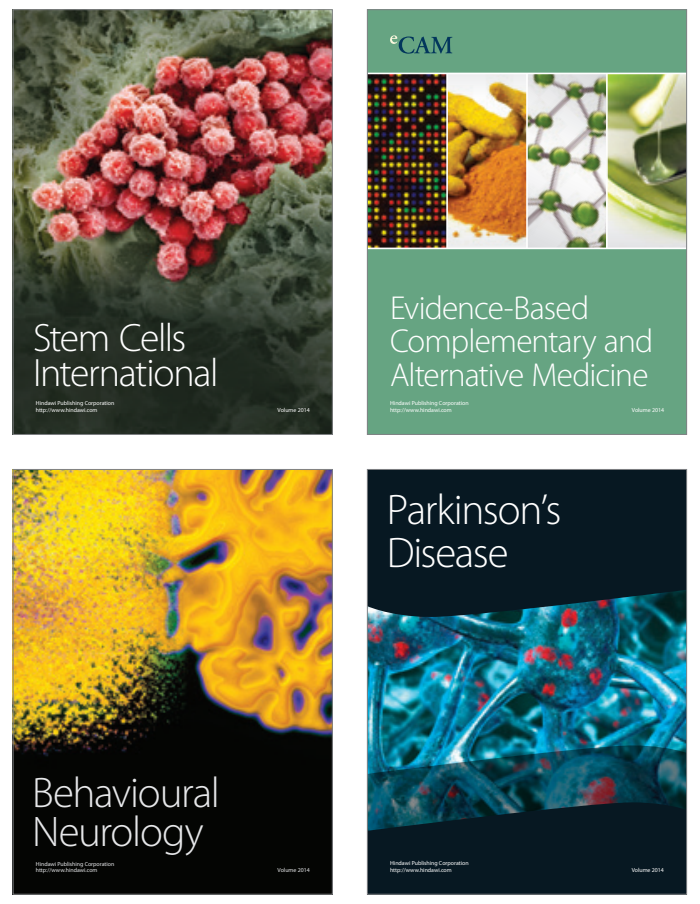
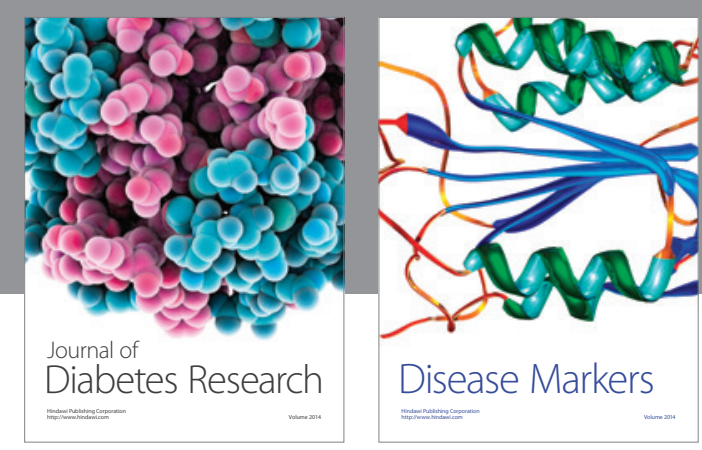

Disease Markers
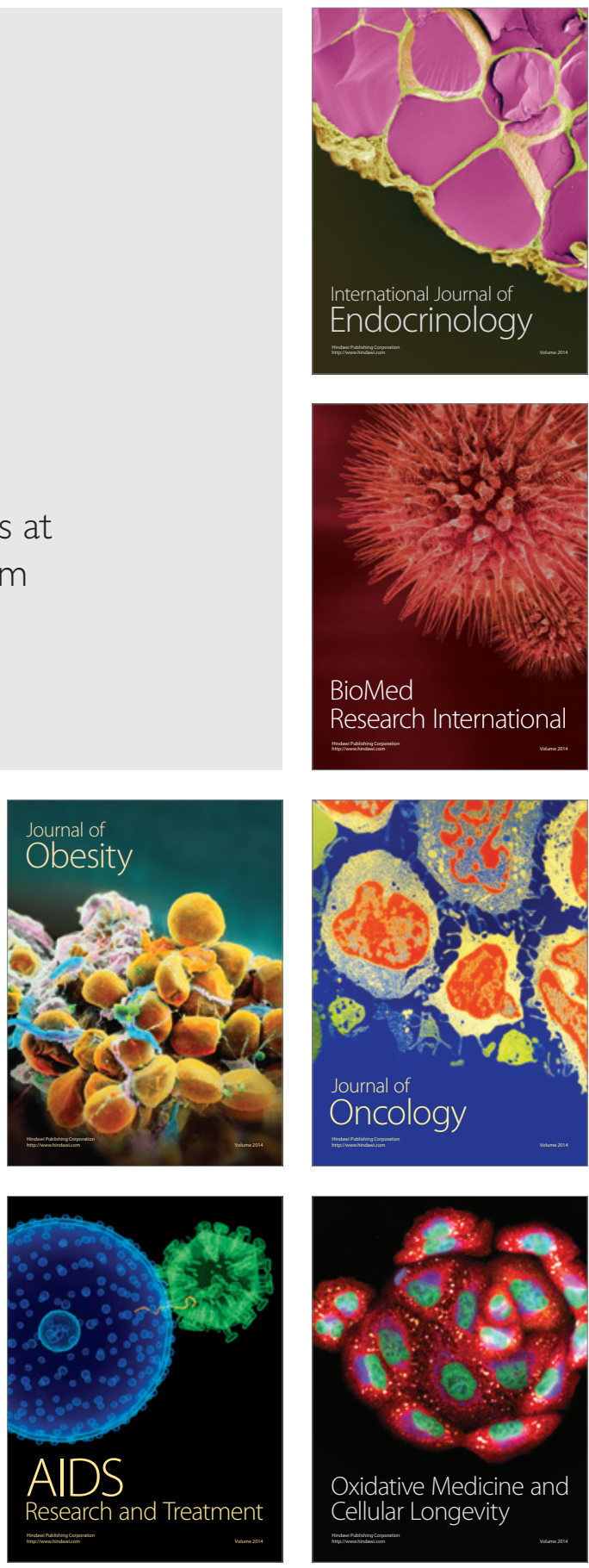